\title{
High c-MET expression is frequent but not associated with early PSA recurrence in prostate cancer
}

\author{
FRANK JACOBSEN $^{1 *}$, SHARAD NOURAIE ASHTIANI $^{1 *}$, PIERRE TENNSTEDT $^{2}$, HANS HEINZER $^{2}$, \\ RONALD SIMON $^{1}$, GUIDO SAUTER ${ }^{1}$, HÜSEYIN SIRMA ${ }^{1}$, MARIA CHRISTINA TSOURLAKIS ${ }^{1}$, \\ SARAH MINNER ${ }^{1}$, THORSTEN SCHLOMM ${ }^{2}$ and UWE MICHL $^{2}$ \\ ${ }^{1}$ Institute of Pathology; ${ }^{2}$ Martini-Clinic Prostate Cancer Center and Section for Translational Prostate Cancer Research \\ at the Clinic of Urology, University Medical Center Hamburg-Eppendorf, D-20246 Hamburg, Germany
}

Received April 26, 2012; Accepted September 14, 2012

DOI: $10.3892 /$ etm.2012.764

\begin{abstract}
MET is considered a possible therapeutic target in numerous tumor types and is also a candidate regulator of response to anti-HER2 and anti-epidermal growth factor receptor (EGFR) therapy. The aim of this study was to determine the prevalence and clinical significance of c-MET expression in hormone-naïve prostate cancers. A pre-existing prostate tissue microarray (TMA) containing samples of 4,177 patients treated by radical prostatectomy was used. A total of 3,378 different prostate cancers were successfully analyzed for c-MET expression by immunohistochemistry and follow-up data were available for 4,104 patients. Membranous c-MET immunostaining was performed for 2,655 (78.6\%) tumors. High c-MET protein expression was significantly associated with a high Gleason grade $(\mathrm{P}=0.0018)$. However, c-MET was not a prognostic marker for biochemical recurrence. c-MET levels were also not associated with other parameters, including tumor stage, nodal stage and surgical margin status. The c-MET protein is often overexpressed in prostate cancer, but has no prognostic relevance. However, the frequent presence of high levels of membranous c-MET protein in prostate cancer cells makes c-MET an attractive target for imaging and treatment.
\end{abstract}

\section{Introduction}

The receptor protein kinase MET was originally identified as an activated oncogene (1), with hepatocyte growth factor (HGF), also known as scatter factor (SF), as the primary ligand. Usually MET is expressed in a variety of epithelial and mesenchymal cells. MET and HGF have been known to induce cell proliferation,

Correspondence to: Dr Uwe Michl, Martini-Clinic Prostate Cancer Center, University Medical Center Hamburg-Eppendorf, Martinistrasse 52, D-20246 Hamburg, Germany

E-mail: michl@martini-klinik.de

${ }^{*}$ Contributed equally

Key words: c-MET, prostate cancer angiogenesis, cell adhesion, invasion, motility and anti-apoptotic responses (2,3). Previous studies have demonstrated a significant MET overexpression in numerous tumor types including glioblastoma (4), melanoma (5), colorectal (6), breast (7), lung (8), gastric (9), thyroid (10) and prostate cancer (11). In addition, aberrant MET expression has been found to be associated with a poor prognosis in a variety of cancers $(4,12)$.

MET expression occurs in the normal prostate epithelium. In contrast to luminal cells, basal cells show consistently high c-MET expression (13). Data on MET expression in prostate cancer are conflicting. Certain studies have reported no associations of high c-MET levels with Gleason grade, while others have reported high MET protein expression in more advanced or metastatic prostate cancer $(11,13-15)$. Recently, c-MET has been proposed as a candidate for targeted cancer therapy in prostate cancer and other tumors (16). In addition, c-MET has been proposed to be a regulator of response to anti-HER 2 and anti-epidermal growth factor receptor (EGFR) therapy. For example, in one study, 64 HER2-positive breast cancer patients with increased c-MET expression demonstrated a decreased response to EGFR/HER2 inhibitor therapy (17). Although the data are controversial and mostly negative, the use of antiEGFR/HER2 therapy remains under discussion for prostate cancer (18).

The aim of this study was to clarify the prevalence and prognostic role of c-MET expression in prostate cancer by using a pre-existing tissue microarray (TMA) including more than 4,000 prostate cancers, the majority with clinical follow-up data. The data show an abundant expression of c-MET and demonstrate that c-MET protein analysis does not serve as a prognostic marker for prostate cancer patients.

\section{Patients and methods}

Patients. A pre-existing prostate cancer TMA consisting of tissue samples from radical prostatectomy specimens of 4,177 patients, consecutively treated at the Department of Urology and the Martini Clinic at the University Medical Center Hamburg-Eppendorf (Hamburg, Germany) between 1997 and 2008 (Table I), was used in this study. Follow-up data were available for 4,104 patients, ranging from 1 to 150 months (mean, 51 months). None of the patients received neo-adjuvant 
or adjuvant therapy. Additional (salvage) therapy was only initiated after biochemical relapse (BCR), the clinical end-point of our study. Prostate-specific antigen (PSA) levels were measured quarterly in the first year, followed by biannual measurements in the second and annual measurements after the third year following surgery. Recurrence was defined as a post-operative PSA of $0.2 \mathrm{ng} / \mathrm{ml}$ and subsequent increase. The first PSA level above or equal to $0.2 \mathrm{ng} / \mathrm{ml}$ was used to define the time of recurrence. Patients without evidence of tumor recurrence were censored at the last follow-up. All prostatectomy specimens were analyzed according to a standard procedure. All prostates were completely paraffin-embedded, including whole-mount sections as previously described (19). A 0.6-mm tissue core was punched out from each sample and transferred to a TMA format as previously described (20). The 4,177 cores were distributed among 9 TMA blocks each containing 129-522 tumor samples. Each TMA block also contained various control tissues including normal prostate tissue and other normal tissues. The utilization of tissues and clinical data was in accordance with the Hamburger Krankenhaus Gesetz ( $\$ 12$ HmbKHG) and approved by our local ethics committee. According to this reputation, informed consent of individual patients was not necessary.

Immunohistochemistry. Freshly cut TMA sections were stained on 1 day in a single experiment. High-temperature pre-treatment of slides was performed in an autoclave in citrate buffer, pH 7.8 for 5 min. c-MET immunostaining was performed using a monoclonal antibody (clone: EP1454, Abcam, Cambridge, UK, dilution 1:150). The Envision system (Dako, Glostrup, Denmark) was used to visualize the immunostaining. Only membranous staining was evaluated as cytoplasmatic staining, if present, was always linked with stronger membranous staining. The staining intensity $(0,1+, 2+, 3+)$ and the fraction of positive tumor cells were recorded for each tissue sample. A final score was created from these 2 parameters according to the following scores: negative scores had a staining intensity of 0 ; weak scores had a staining intensity of $1+$ in $\leq 70 \%$ of tumor cells or a staining intensity of $2+$ in $\leq 30 \%$ of tumor cells; moderate scores had a staining intensity of $1+$ in $>70 \%$ of tumor cells or a staining intensity of $2+$ in $>30 \%$ but $\leq 70 \%$ of tumor cells or a staining intensity of $3+$ in $\leq 30 \%$ of tumor cells; and strong scores had a staining intensity of $2+$ in $>70 \%$ of tumor cells or a staining intensity of $3+$ in $>30 \%$ of tumor cells.

Statistic analysis. For statistical analysis, the JMP 8.0 software (SAS Institute Inc., Cary, NC, USA) was used. Contingency tables were calculated to determine the association between the c-MET immunostaining score and clinicopathological variables. The Chi-square test was used to identify significant associations. Kaplan-Meier curves were generated for PSA recurrence-free survival. The log-rank test was performed to determine the significance of differences between stratified survival functions. Cox proportional hazards regression analysis was performed to determine the statistical independence and significance between pathological, molecular and clinical variables.

\section{Results}

Technical issues. A total of 3,378 (81.6\%) tumor samples were successfully analyzed in our TMA analysis. The reasons for
Table I. Pathological and clinical data of the arrayed prostate cancers.

\begin{tabular}{|c|c|c|}
\hline \multirow[b]{2}{*}{ Variable } & \multicolumn{2}{|c|}{ No. of patients ${ }^{\mathrm{a}}$} \\
\hline & $\begin{array}{l}\text { Study cohort } \\
\text { on TMA } \\
(n=4,177)\end{array}$ & $\begin{array}{c}\text { Biochemical relapse } \\
\text { among categories } \\
(n=4,104)\end{array}$ \\
\hline \multicolumn{3}{|c|}{ Follow-up (months) } \\
\hline Mean & 51.1 & - \\
\hline Median & 38.1 & - \\
\hline \multicolumn{3}{|c|}{ Age (years) } \\
\hline$<50$ & 119 & 119 \\
\hline $50-60$ & 1,249 & 1,237 \\
\hline $60-70$ & 2,388 & 2,347 \\
\hline$>70$ & 277 & 260 \\
\hline \multicolumn{3}{|c|}{ Pre-treatment PSA (ng/ml) } \\
\hline$<4$ & 631 & 625 \\
\hline $4-10$ & 2,356 & 2,230 \\
\hline $10-20$ & 774 & 759 \\
\hline$>20$ & 225 & 203 \\
\hline \multicolumn{3}{|c|}{ pT category (AJCC 2002) } \\
\hline pT2 & 2,789 & 2,780 \\
\hline pT3a & 806 & 786 \\
\hline pT3b & 412 & 374 \\
\hline pT4 & 25 & 33 \\
\hline \multicolumn{3}{|c|}{ Gleason grade } \\
\hline$\leq 3+3$ & 1,593 & 1,589 \\
\hline $3+4$ & 1,847 & 1,828 \\
\hline $4+3$ & 442 & 426 \\
\hline$\geq 4+4$ & 146 & 115 \\
\hline \multicolumn{3}{|c|}{ pN category } \\
\hline pNO & 1,882 & 1,840 \\
\hline $\mathrm{pN}+$ & 146 & 123 \\
\hline \multicolumn{3}{|c|}{ Surgical margin } \\
\hline Negative & 3,255 & 3,224 \\
\hline Positive & 751 & 717 \\
\hline
\end{tabular}

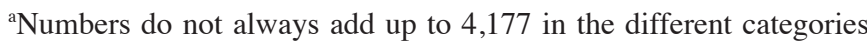
due to cases with missing data. TMA, tissue microarray; PSA, prostate-specific antigen; AJCC, American Joint Commission of Cancer staging system.

non-informative cases $(762 ; 19.4 \%)$ included a lack of tissue samples or absence of unequivocal cancer tissue in individual TMA samples.

Immunohistochemistry. c-MET immunostaining revealed strong membrane staining in our tissues. Although certain cytoplasmic staining was sometimes observed, this was always associated with a markedly higher staining level at the membranes. Membranous c-MET immunostaining was recorded in 2,655 (78.6\%) of 3,378 successfully analyzed cases. Staining was weak in 780 (23.1\%), moderate in $801(23.7 \%)$ and strong in 1074 (31.8\%) prostate cancers (Fig. 1B and C). In benign prostate epithelium, c-MET was always strongly 
Table II. MET expression and tumor phenotype.

\begin{tabular}{|c|c|c|c|c|c|c|}
\hline \multirow[b]{2}{*}{ Variable } & \multirow{2}{*}{$\begin{array}{l}\text { No. of successfully } \\
\text { analyzed samples }\end{array}$} & \multicolumn{4}{|c|}{ c-MET immunohistochemistry result } & \multirow[b]{2}{*}{ P-value } \\
\hline & & Negative (\%) & Weak $(\%)$ & Moderate (\%) & Strong $(\%)$ & \\
\hline All tumors & $3,378^{\mathrm{a}}$ & 21.4 & 23.1 & 23.7 & 31.8 & \\
\hline \multicolumn{7}{|c|}{ Tumor stage } \\
\hline pT2 & 2,203 & 22.2 & 23.5 & 22.9 & 31.4 & 0.1191 \\
\hline pT3a & 695 & 20.3 & 20.4 & 25.0 & 34.2 & \\
\hline pT3b & 335 & 20.0 & 26.0 & 23.9 & 30.1 & \\
\hline pT4 & 21 & 4.8 & 23.8 & 19.0 & 52.4 & \\
\hline \multicolumn{7}{|c|}{ Gleason grade } \\
\hline$\leq 3+3$ & 1,204 & 24.1 & 25.0 & 21.2 & 29.7 & 0.0018 \\
\hline $3+4$ & 1,567 & 20.9 & 21.6 & 25.1 & 32.4 & \\
\hline $4+3$ & 362 & 16.6 & 22.7 & 26.0 & 34.8 & \\
\hline$\geq 4+4$ & 118 & 16.9 & 26.3 & 16.9 & 39.8 & \\
\hline \multicolumn{7}{|l|}{ Nodal stage } \\
\hline No & 1,529 & 18.3 & 21.9 & 24.3 & 35.5 & 0.3907 \\
\hline $\mathrm{N}+$ & 123 & 23.6 & 23.6 & 19.5 & 33.3 & \\
\hline \multicolumn{7}{|c|}{ Surgical margin } \\
\hline Negative & 2,602 & 21.4 & 23.5 & 23.3 & 31.8 & 0.758 \\
\hline Positive & 632 & 21.8 & 21.5 & 24.4 & 32.3 & \\
\hline \multicolumn{7}{|c|}{ Pre-operative PSA level (ng/ml) } \\
\hline$<4$ & 468 & 16.9 & 22.4 & 25.4 & 35.3 & 0.0064 \\
\hline $4-10$ & 1,918 & 20.9 & 22.7 & 24.7 & 31.8 & \\
\hline $10-20$ & 645 & 25.6 & 24.3 & 20.6 & 29.5 & \\
\hline$>20$ & 191 & 26.7 & 22.5 & 17.8 & 33.0 & \\
\hline
\end{tabular}

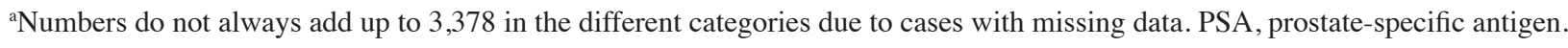

expressed in prostate basal cells (Fig. 1E and F), with a weaker expression in luminal cells as compared with the majority of invasive cancers (Fig. 1A-D). The association between c-MET immunostaining and tumor phenotype is summarized in Table II. Significant associations were demonstrated between strong c-MET expression and high Gleason grade $(\mathrm{P}=0.0018)$ as well as increased pre-operative PSA levels $(\mathrm{P}=0.0064)$. c-MET staining levels were not associated with tumor stage $(\mathrm{P}=0.1191)$, nodal stage $(\mathrm{P}=0.3907)$, surgical margin status $(\mathrm{P}=0.758)$ or pre-operative PSA level $(\mathrm{P}=0.0064)$. Follow-up data were available for 4,104 patients and 3,378 cases were successfully analyzed. As predicted, Gleason grade and pT stage were significantly associated with PSA recurrence in this patient subset $(\mathrm{P}<0.0001$ each; Fig. $2 \mathrm{~A}$ and $\mathrm{B})$. However, c-MET protein expression levels were not associated with the risk of PSA recurrence ( $\mathrm{P}=0.949$, Fig. $2 \mathrm{C})$.

\section{Discussion}

In the present study, the frequency and the potential clinicopathological role of c-MET protein expression was investigated in prostate cancer. The data demonstrate that c-MET expression is abundant in prostate cancer but lacks a clear association with an unfavorable phenotype or a poor clinical outcome.
Membranous c-MET staining was observed in 2,655 (78.6\%) of 3,378 successfully analyzed cancers and strong c-MET expression was significantly associated with a high Gleason grade $(\mathrm{P}=0.0018)$. These results are within the range of previous studies using immunohistochemistry, although the reported c-MET expression levels vary from 33 to $84 \%$ (11,13-15). However, the number of analyzed tumors was markedly lower in these studies compared with the present cohort. Pisters et al (15) analyzed a cohort of 43 primary prostate cancers. They observed c-MET expression in $84 \%$ of cases and revealed an association between c-MET expression and advanced grade prostate cancers $(\mathrm{P}<0.001)$. Another group examined 108 prostate cancers and observed c-MET expression in $45 \%$ of cases. Their group distinguished between cytoplasmic or luminal membrane staining. No correlation was observed with Gleason grade (11). Watanabe et al (14) investigated a cohort of 36 patients, $33 \%$ of 'latent' and $71 \%$ of 'clinical significant' prostate cancers displayed cytoplasmic c-MET expression. In total, $38 \%$ of low-grade and $80 \%$ of high-grade prostate cancers presented c-MET expression. Knudsen et al (13) analyzed a cohort of 90 low-grade tumors (Gleason score 6 or 7). c-MET expression was observed in $51 \%$ of cancers. They could not identify any correlation between c-MET expression and disease progression. Overall, these studies demonstrate a wide range of 

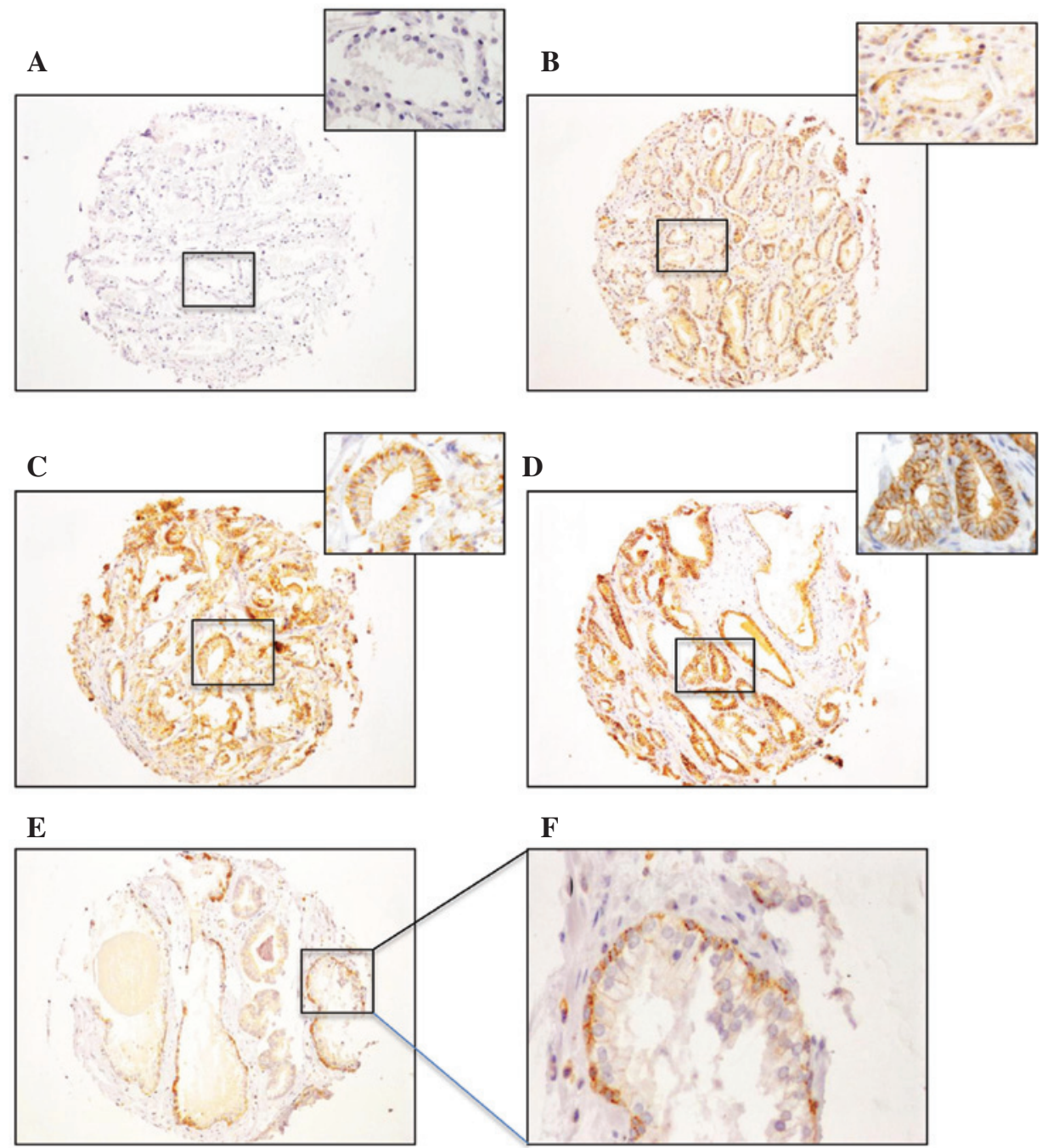

Figure 1. Examples of c-Met immunostaining in prostate cancer and normal tissues. (A) Lack of staining; (B) weak membranous staining; (C) moderate membranous staining; (D) strong membranous staining; (E) strong membranous staining in basal cells; (F) magnification of (E).

A

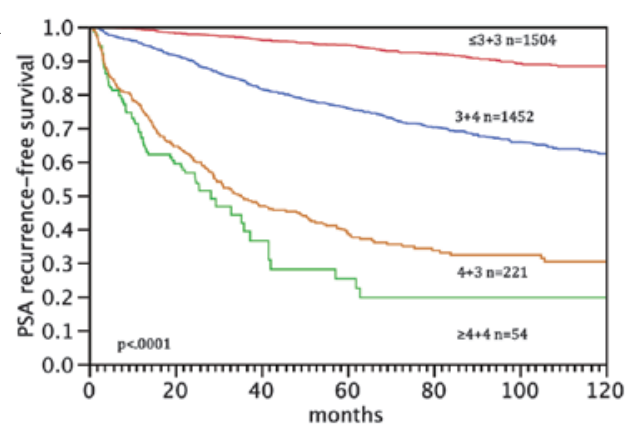

B

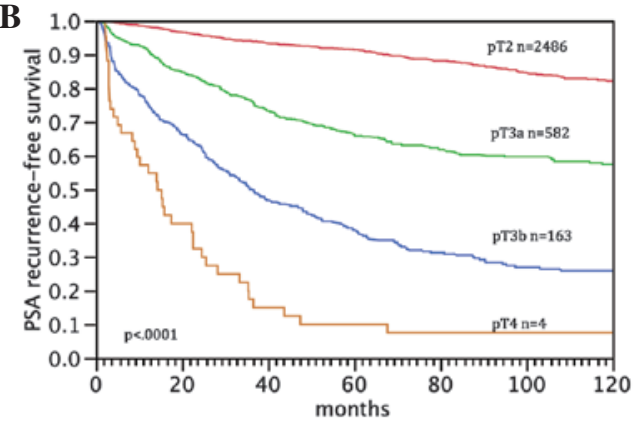

C

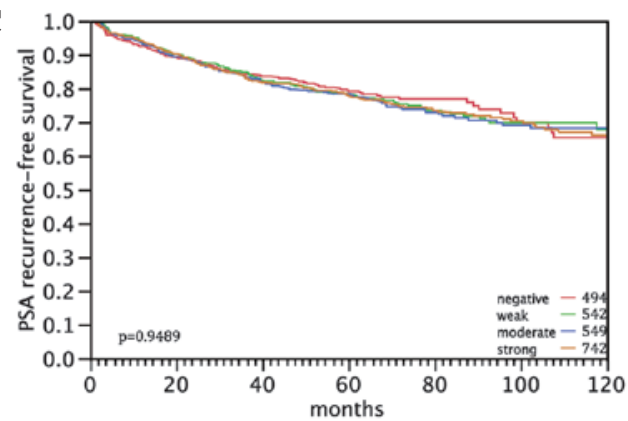

Figure 2. Influence of clinicopathological features and c-MET staining on PSA recurrence. (A) Gleason grade. (B) pT category. (C) c-MET immunostaining. PSA, prostate-specific antigen. 
c-MET expression rates and are not always consistent with our findings.

It is possible that these controversial data are partly attributable to sampling issues as these studies have all analyzed markedly small patient cohorts and are characterized by highly variable definitions of c-MET-positivity. Watanabe et al (14) considered a tumor sample as positive if more than $30 \%$ of the tumor cells stained for c-MET, while Humphrey et al required only more than $5 \%$ to classify a tumor as positive (11). Knudsen et al (13) evaluated at least moderate staining intensity as c-MET positive. In addition, the staining of the secretory cells differs from the previous described studies. In the report by Pisters et al (15), secretory cell c-MET expression is limited to the central zone. Other studies do not note regional variation of expression $(11,14)$. In contrast Knudsen et al (13) did not observe expression of c-MET in secretory cells.

c-MET is frequently expressed in a variety of other cancers. For some cancers, including cholangiocarcinoma, gastric or skin cancer, a clear correlation between c-MET expression level and a poor prognosis has been demonstrated $(5,21,22)$. A skin cancer study revealed significant overexpression of c-MET in all skin cancers with stronger positive responce in malignant melanomas. c-MET expression was stronger in deeper melanomas than in superficial ones (5). However, other investigations identified no significant association between c-MET expression level and clinicopathological parameters (23). Accordingly c-MET could only be used as a prognostic marker in certain cancer types, but not in others.

The high frequency of expression in prostate and other cancer types makes c-MET an attractive potential therapeutic target. Recently, several studies with c-MET inhibitors were realized or are in progress $(24,25)$. Recently published studies demonstrate the anti-proliferative efficacy of c-MET inhibitors in combination with androgen ablation therapy for advanced prostate cancer $(16,17)$. This illustrates that co-targeting of c-MET and androgen signaling pathway might be a therapeutic option for the treatment of prostate cancer in the future (16).

In conclusion, the results of this study reveal that c-MET is frequently overexpressed in prostate cancer. A significant correlation was demonstrated between strong c-MET expression and high Gleason grade, but not with other clinicopathological parameters. Although c-MET appears to be involved in the progression of prostate cancer, this study does not confirm a role of c-MET as a prognostic marker in patients with prostate cancer.

\section{References}

1. Cooper CS, Park M, Blair DG, et al: Molecular cloning of a new transforming gene from a chemically transformed human cell line. Nature 311: 29-33, 1984.

2. Knudsen BS and Edlund M: Prostate cancer and the met hepatocyte growth factor receptor. Adv Cancer Res 91: 31-67, 2004.

3. Peruzzi B and Bottaro DP: Targeting the c-Met signaling pathway in cancer. Clin Cancer Res 12: 3657-3660, 2006.

4. Kong DS, Song SY, Kim DH, et al: Prognostic significance of c-Met expression in glioblastomas. Cancer 115: 140-148, 2009.
5. Lee YJ, Kim DH, Lee SH, Kim DW, Nam HS and Cho MK: Expression of the c-Met proteins in palignant skin cancers. Ann Dermatol 23: 33-38, 2011.

6. De Oliveira AT, Matos D, Logullo AF, et al: MET Is highly expressed in advanced stages of colorectal cancer and indicates worse prognosis and mortality. Anticancer Res 29: 4807-4811, 2009.

7. Matteucci E, Bendinelli P and Desiderio MA: Nuclear localization of active HGF receptor Met in aggressive MDA-MB231 breast carcinoma cells. Carcinogenesis 30: 937-945, 2009.

8. Ichimura E, Maeshima A, Nakajima T and Nakamura T: Expression of c-met/HGF receptor in human non-small cell lung carcinomas in vitro and in vivo and its prognostic significance. Jpn J Cancer Res 87: 1063-1069, 1996.

9. Kuniyasu H, Yasui W, Yokozaki H, Kitadai Y and Tahara E: Aberrant expression of c-met mRNA in human gastric carcinomas. Int J Cancer 55: 72-75, 1993.

10. Belfiore A, Gangemi P, Costantino A, et al: Negative/low expression of the Met/hepatocyte growth factor receptor identifies papillary thyroid carcinomas with high risk of distant metastases. J Clin Endocrinol Metab 82: 2322-2328, 1997.

11. Humphrey PA, Zhu X, Zarnegar R, et al: Hepatocyte growth factor and its receptor (c-MET) in prostatic carcinoma. Am J Pathol 147: 386-396, 1995.

12. Birchmeier C, Birchmeier W, Gherardi E and Vande Woude GF: Met, metastasis, motility and more. Nat Rev Mol Cell Biol 4: 915-925, 2003.

13. Knudsen BS, Gmyrek GA, Inra J, et al: High expression of the Met receptor in prostate cancer metastasis to bone. Urology 60: 1113-1117, 2002.

14. Watanabe M, Fukutome K, Kato H, et al: Progression-linked overexpression of c-Met in prostatic intraepithelial neoplasia and latent as well as clinical prostate cancers. Cancer Lett 141: 173-178, 1999.

15. Pisters LL, Troncoso P, Zhau HE, Li W, von Eschenbach AC and Chung LW: c-met proto-oncogene expression in benign and malignant human prostate tissues. J Urol 154: 293-298, 1995.

16. Tu WH, Zhu C, Clark C, Christensen JG and Sun Z: Efficacy of c-Met inhibitor for advanced prostate cancer. BMC Cancer 10: 556,2010

17. Varkaris A, Corn PG, Gaur S, Dayyani F, Logothetis CJ and Gallick GE: The role of $\mathrm{HGF} / \mathrm{c}-$ Met signaling in prostate cancer progression and c-Met inhibitors in clinical trials. Expert Opin Investig Drugs 20: 1677-1684, 2011.

18. Gross ME, Jo S and Agus DB: Update on HER-kinase-directed therapy in prostate cancer. Clin Adv Hematol Oncol 2: 53-56, $64,2004$.

19. Erbersdobler A, Fritz H, Schnöger S, et al: Tumour grade, proliferation, apoptosis, microvessel density, $\mathrm{p} 53$, and bcl-2 in prostate cancers: differences between tumours located in the transition zone and in the peripheral zone. Eur Urol 41: 40-46, 2002.

20. Bubendorf L: High-throughput microarray technologies: from genomics to clinics. Eur Urol 40: 231-238, 2001.

21. Miyamoto M, Ojima H, Iwasaki M, et al: Prognostic significance of overexpression of c-Met oncoprotein in cholangiocarcinoma. Br J Cancer 105: 131-138, 2011.

22. Amemiya H, Menolascino $F$ and Peña A: Role of the expression of c-Met receptor in the progression of gastric cancer. Invest Clin 51: 369-380, 2010 (In Spanish).

23. Freudlsperger C, Alexander D, Reinert S and Hoffmann J: Prognostic value of c-Met expression in oral squamous cell carcinoma. Exp Ther Med 1: 69-72, 2010.

24. Previdi S, Abbadessa G, Dalò F, France DS and Broggini M: Breast cancer-derived bone metastasis can be effectively reduced through specific c-MET inhibitor tivantinib (ARQ 197) and shRNA c-MET knockdown. Mol Cancer Ther 11: 214-223, 2012.

25. Liu X, Wang Q, Yang G, et al: A novel kinase inhibitor, INCB28060, blocks c-MET-dependent signaling, neoplastic activities, and cross-talk with EGFR and HER-3. Clin Cancer Res 17: 7127-7138, 2011 